\title{
The detection of stellar velocity dispersion drops in the central regions of five isolated Seyfert spirals ${ }^{\star}$
}

\author{
I. Márquez ${ }^{1}$, J. Masegosa ${ }^{1}$, F. Durret ${ }^{2}$, R. M. González Delgado ${ }^{1}$, M. Moles ${ }^{1}$, J. Maza ${ }^{3}$, E. Pérez ${ }^{1}$, and M. Roth ${ }^{4}$ \\ 1 Instituto de Astrofísica de Andalucía (CSIC), Apartado 3004, 18080 Granada, Spain \\ 2 Institut d'Astrophysique de Paris, CNRS, 98bis Bld Arago, 75014 Paris, France \\ 3 Departamento de Astronomía, Universidad de Chile, Casilla 36D, Santiago, Chile \\ ${ }^{4}$ Observatories of the Carnegie Institution of Washington, 813 Barbara Street, Pasadena, CA91101, USA
}

Received 7 March 2003 / Accepted 25 June 2003

\begin{abstract}
We analyze the kinematics of the central regions of five isolated Seyfert spiral galaxies from the DEGAS sample (four with new data presented in this paper, IC 184, UGC 3223, NGC 2639, NGC 6814, and NGC 6951 from our previous data), by using long slit spectroscopy in the CaII triplet range (at $\approx 8600 \AA$ ) obtained with a 4 m-class telescope. A drop of the velocity dispersions in the innermost $\pm(1-3)$ arcsec is observed in four of them, and hinted at in the remaining galaxy (NGC 6814). The available HST images for our sample together with another nine galaxies with reported velocity dispersion drops are also used to investigate the presence of morphological inner structures at the scales of the kinematical drops. Evidence for disk-like shapes is found in 12 out of the 14 cases. The only exceptions are NGC 6814 and NGC 6951. Existing N-body simulations including stars, gas and star formation predict that such a drop is most probably due to a young stellar population born from dynamically cold gas accreted in a circumnuclear disk formed during an episode of central gas accretion driven by a bar. The equivalent widths of the calcium triplet lines for our 5 galaxies have been measured. Even if the profiles could be formally consistent with constant $E W(\mathrm{CaT})$ values, they seem to indicate the presence of a local maximum in the regions corresponding spatially to the drops; if confirmed, this would imply the presence of a different stellar population, whose properties could help constrain the models.
\end{abstract}

Key words. galaxies: spiral - galaxies: kinematics and dynamics - galaxies: structure - galaxies: interactions

\section{Introduction}

The existence of non-axisymmetric components of the galactic potential has been frequently invoked as an efficient way to transport gas from the galaxy scale down to the nucleus to fuel the active galactic nucleus (AGN). In particular, the shocks and gravitational torques induced by a galactic bar provide the non axisymmetric potential invoked in theoretical works (Simkin et al. 1980; Shlosman et al. 1989; Barnes \& Hernquist 1991) to make the gas lose angular momentum and therefore facilitate the fueling mechanism. Whether there is no preference for Seyfert nuclei to occur in barred galaxies (Heckman 1980; Simkim et al. 1980; Moles et al. 1995; McLeod \& Rieke 1995; Mulchaey \& Regan 1997) or whether an excess of bars among Seyferts exists, is still an open issue. Nevertheless, in addition to the large scale bar, another mechanism is needed

Send offprint requests to: I. Márquez, e-mail: isabel@iaa.es

* Based on observations made with the WHT operated on the island of La Palma by ING in the Spanish Observatorio del Roque de Los Muchachos of the Instituto de Astrofísica de Canarias. to drive the gas to the very central regions; one such mechanism is that of nested bars (Shlosman et al. 1989; Friedli \& Martinet 1993; Combes 1994; Heller \& Shlosman 1994), which has been suggested to fuel molecular gas into an intense central starburst in NGC 2782 (Jogee et al. 1999) and in a Seyfert 2 galaxy, Circinus (Maiolino et al. 2000). However, the analysis of the HST images of Seyferts (Regan \& Mulchaey 1999; Martini \& Pogge 1999; Pérez et al. 2000; Martini et al. 2003a,b) has shown that such nuclear bars are not ubiquitous, and nuclear spirals are proposed to be one of the channels to feed gas into the central engine.

Stellar kinematical profiles of four double barred galaxy hosts of AGNs obtained with the VLT and ISAAC have recently shown the existence of decoupled kinematics in the nuclear region, as well as a drop of the velocity dispersion in three of them (Emsellem et al. 2001). Although their models could not properly reproduce this drop, Emsellem et al. suggested that it was due to a transient cold nuclear disk fueled by gas inflow along the bar, which has recently formed new stars. Models by Wozniak \& Michel-Dansac (2003) have recently succeeded in accounting for this drop in velocity dispersion, 
which indeed seems to be due to a young stellar population born from dynamically cold gas accreted in a circumnuclear disk. The presence of such disks can be directly related to the circumnuclear structures required to drive the gas to the very central regions, as nuclear (nested) bars or nuclear spirals (see references above).

In most of these studies the environmental effects are in general not considered when studying the properties of Seyfert galaxies and/or comparing them with those of non-active ones (but see Moles et al. 1995). However, the properties of spiral galaxies can be modified even in mild interactions, as shown by Márquez et al. (2002) for a sample of 111 galaxies ranging from truly isolated to mildly interacting spirals. Therefore, we decided to study the properties of isolated Seyfert spirals, and to compare them with a control sample of isolated non-active spirals; the main result is that the active and non-active samples are equivalent in the studied properties: large scale disks, bulges and bars have the same properties, as derived by the analysis of their NIR images (Márquez et al. 1999, 2000). To further delimit eventual differences/similarities and to put some constraints on the possible mechanisms taking place in the circumnuclear regions, kinematical information is needed; therefore, long slit spectra along several position angles were obtained for a number of our sample galaxies, to get both gas (2/3 of the whole sample) and stellar kinematics (for a subsample). The results of the overall kinematical analysis together with the information on metallicities provided by the analysis of the HII regions will be published separately (Márquez et al. 2003). High spectral and spatial resolution spectra in the CaT region have been obtained for a total of eight Seyfert galaxies. In four of them we have found drops in the stellar velocity dispersion of the central regions, together with a hint of such a drop in NGC 6814, comparable to those reported by Emsellem et al. (2001).

Section 2 is devoted to the presentation of the observations, data reduction and analysis. The results of the analysis are reported in Sect. 3, and the discussion and conclusions in Sect. 4.

\section{Observations, data reduction and analysis}

The DEGAS sample includes 33 isolated galaxies, 17 with an AGN and 16 without an AGN taken as a control sample. The four galaxies reported here all have an AGN and their infrared properties have already been described (Márquez et al. 1999). As a complement to these infrared images, we have retrieved the HST images for these four galaxies and present them here. All were taken with the F606W filter.

The spectroscopic data for the gas kinematics were collected with the ISIS Spectrograph at the $4 \mathrm{~m}$ William Herschel Telescope (WHT) in La Palma (Spain). The setup and main characteristics of the observations are given in Table 1. The spectral and spatial samplings were $0.39 \AA /$ pixel and $0.36 \mathrm{arcsec} / \mathrm{pixel}$, respectively, and the slit width 1.03 arcsec. The spectral resolution given in Table 1 has been measured as the FWHM of a Gaussian fit to several unblended sky lines.
Table 1. Detailed log of the spectroscopic observations.

\begin{tabular}{lcrcrrr}
\hline \hline Galaxy & Date & $\begin{array}{r}\text { Spectral } \\
\text { range }(\AA)\end{array}$ & $\begin{array}{r}<\text { Seeing }> \\
(\operatorname{arcsec})\end{array}$ & $\begin{array}{r}\text { PA } \\
\left({ }^{\circ}\right)\end{array}$ & $\begin{array}{r}\text { Exp. } \\
(\mathrm{s})\end{array}$ & $\begin{array}{c}\text { Spect. } \\
\text { resol. }\end{array}$ \\
\hline IC 184 & 1999 & $8476-8872$ & 1.5 & 7 & 7200 & 0.71 \\
& 1999 & $8476-8872$ & 1.5 & 97 & 5400 & 0.71 \\
UGC 3223 & 1999 & $8476-8872$ & 1.5 & 80 & 5400 & 0.71 \\
NGC 2639 & 1999 & $8476-8872$ & 1.5 & 45 & 5400 & 0.68 \\
& 1999 & $8476-8872$ & 1.5 & 135 & 5400 & 0.71 \\
NGC 6814 & 1996 & $8505-8881$ & 1.2 & 30 & 3600 & 0.71 \\
& 1996 & $8505-8881$ & 1.2 & 120 & 5400 & 0.78 \\
\hline
\end{tabular}

Standard IRAF ${ }^{1}$ procedures were used for the reduction of the spectroscopic data, following the standard steps of bias subtraction, flat field correction, wavelength calibration with a CuNe lamp observed before and after the target, atmospheric extinction correction, and flux calibration using spectroscopic standards observed through an 8 arcsec wide slit. The sky background level was determined taking median averages over two strips on either side of the galaxy signal. We used crosscorrelation techniques as described in Pérez et al. (2000) and references therein, to extract the kinematic information, using as templates for the cross-correlation the observed spectra of several stars. The errors refer to the dispersion in the velocity shifts measured with respect to the different template stars. For clarity, error bars in the stellar velocity distributions are not plotted, but they typically amount to $\pm 50 \mathrm{~km} \mathrm{~s}^{-1}$, with median values of $\pm 35 \mathrm{~km} \mathrm{~s}^{-1}$ for NGC 6951 , and up to $\pm 70 \mathrm{~km} \mathrm{~s}^{-1}$ for NGC 2639. The systemic velocity has been chosen as that of the section with the maximum continuum level, except for IC 184, for which the kinematical center (the point that allows the best symmetrization of the rotation curve) is shifted by about 0.8 arcsec to the South with respect to the continuum maximum.

The calcium triplet equivalent width has been measured following the method described by Terlevich et al. (1990, hereafter, TDT90), which uses two specific continuum bands on either side of the CaT region, after Doppler shift corrections for each section. Due to a shorter spectral range coverage than TDT90, slight modifications of the central wavelength of the "blue" continuum were adopted for all the galaxies except NGC 2639. For the "red" continuum, we had to use much shorter wavelengths than TDT90. The resulting equivalent widths for the inner regions of each galaxy (see Sect. 3) are given in Table 3 . In the last two columns the central rest frame wavelength of each continuum band is given (the width used is $15 \AA$ ). The differences in the continuum bands used may be the reason why we measure systematically lower equivalent widths $(E W)$ than those reported in TDT90 for the same types of active galaxies. As TDT90 pointed out, to obtain conclusions on the stellar population based on CaII triplet measurements, exactly the same method has to be used. For our data the results are specially tricky due to the fact that the TiO and VO bands contaminate the "red" continuum. In order to

${ }^{1}$ IRAF is the Image Analysis and Reduction Facility made available to the astronomical community by the National Optical Astronomy Observatories, which are operated by the Association of Universities for Research in Astronomy (AURA), Inc., under contract with the U.S. National Science Foundation. 
Table 2. Details on HST retrieved images.

\begin{tabular}{lllrcc}
\hline \hline Galaxy & AGN* & Band & $\begin{array}{c}\text { Exp. } \\
(\mathrm{s})\end{array}$ & $\begin{array}{c}\text { Prog. } \\
\text { Nr. }\end{array}$ & PI \\
\hline IC 184 & Sy2 & F606W & 500 & 5479 & M. Malkan \\
UGC 3223 & Sy1 & F606W & 500 & 5479 & M. Malkan \\
NGC 2639 & Sy1.9 & F606W & 500 & 5479 & M. Malkan \\
NGC 6814 & Sy1.5 & F606W & 500 & 5479 & M. Malkan \\
NGC 1395 & Sy1.8 & F606W & 1680 & 8597 & M. Regan \\
NGC 1808 & Sy2 & F814W & 160 & 6872 & J. Flood \\
NGC 3412 & - & F606W & 160 & 5446 & G. Illingworth \\
NGC 3623 & L=LINER & F606W & 160 & 5446 & G. Illingworth \\
NGC 3627 & L/Sy2 & F606W & 560 & 8597 & M. Regan \\
NGC 4303 & Sy2 & F814W & 460 & 9042 & S. Smartt \\
NGC 4477 & Sy2 & F606W & 160 & 5446 & G. Illingworth \\
NGC 4579 & L/Sy1.9 & F658N & 1400 & 6436 & D. Maoz \\
NGC 4725** & Sy2 & F606W & 560 & 8597 & M. Regan \\
NGC 6503 & L & F814W & 2600 & 8602 & A. Filippenko \\
\hline
\end{tabular}

* Taken from NED.

** Diffraction spikes impede a reliable determination of the hinted central elongated structure.

quantify the uncertainty due to the choice of the continuum level, we have used three different "red" continua and measured the corresponding $E W$; the error bars in Fig. 6 are calculated as the dispersion of these three values, and therefore represent lower limits to the true error bars. The minimum uncertainty obtained for the best cases with a flat continuum is $0.5 \AA$ but in most of the galaxies the uncertainty can be larger than $1.5 \AA$.

$\mathrm{CaT}$ equivalent widths have also been measured using the method of Cenarro et al. (2001). The comparison between this method and that of TDT90 has shown that, in the case of dilution due to an important underlying old stellar population, TDT90 seem to be less sensitive to the contamination by TiO and VO lines. Therefore, since our sample galaxies are all early type spirals, we have adopted the TDT90 method.

For our five sample galaxies and for nine of the eleven galaxies with velocity dispersion drops reported in the literature (see Sect. 4) the available archival WFPC2 $\mathrm{HST}^{2}$ optical images (see Table 2 for details) have been used to identify features which, according to numerical models, could possibly be related to the kinematics. To trace the presence of morphological features in the innermost few arcseconds, both raw and sharp-divided images ${ }^{3}$ have been used. Sharp-divided images are very useful to trace asymmetries in the light distribution, such as bars, spiral arms, dust lanes and rings. This technique allows the subtraction of the diffuse background in a very convenient way to look for subtle, small-scale variations and discuss the possible presence of both dust extinguished and more luminous regions (Sofue et al. 1994; Márquez \& Moles 1996; Erwin \& Sparke 1999; Laine et al. 1999; Márquez et al. 1999; Erwin \& Sparke 2002).

\footnotetext{
${ }^{2}$ Based on observations collected with the NASA/ESA HST, obtained at STScI which is operated by AURA Inc., under NASA contract NAS5-26555.

3 The result of dividing the original images by medianfiltered ones.
}

Table 3. CaT equivalent widths.

\begin{tabular}{lrccccc}
\hline \hline Galaxy & PA & $\begin{array}{c}E W \\
\mathrm{Ca} 2\end{array}$ & $\begin{array}{c}E W \\
\mathrm{Ca} 3\end{array}$ & $\begin{array}{c}E W \\
\text { CaII }\end{array}$ & $\begin{array}{c}\text { "blue" } \\
\text { cont. }\end{array}$ & $\begin{array}{c}\text { "red" } \\
\text { cont. }\end{array}$ \\
\hline \multirow{2}{*}{ IC 184 } & 7 & $3.5(0.3)$ & $2.8(0.6)$ & $6.3(1.8)$ & 8458 & 8680 \\
& 97 & $4.0(0.3)$ & $2.7(0.4)$ & $6.7(0.6)$ & 8458 & 8680 \\
UGC 3223 & 80 & $3.5(0.1)$ & $2.8(0.3)$ & $6.3(0.7)$ & 8458 & 8700 \\
NGC 2639 & 45 & $3.0(0.1)$ & $2.6(0.1)$ & $5.7(0.3)$ & 8455 & 8700 \\
& 135 & $3.5(0.1)$ & $2.6(0.2)$ & $6.1(0.5)$ & 8455 & 8700 \\
NGC 6814 & 30 & $3.5(0.2)$ & $2.5(0.5)$ & $6.1(1.3)$ & 8484 & 8700 \\
& 120 & $3.5(0.2)$ & $2.5(0.5)$ & $6.0(1.3)$ & 8484 & 8700 \\
NGC 6951 & 48 & $3.7(0.1)$ & $3.3(0.1)$ & $7.0(0.3)$ & 8482 & 8750 \\
& 138 & $3.4(0.1)$ & $3.1(0.3)$ & $6.6(0.7)$ & 8482 & 8750 \\
\hline
\end{tabular}

$\mathrm{Ca} 2=\mathrm{CaT}$ line at $8542 \AA$.

$\mathrm{Ca} 3=\mathrm{CaT}$ line at $8662 \AA$.

$E W(\mathrm{CaII})=E W(\mathrm{Ca} 2)+E W(\mathrm{Ca} 3)$.

\section{Results}

The kinematical data and the images used in the analysis of the data are displayed in Figs. 1 to 4 . The points corresponding to gas kinematics are given for comparison (for further details on the gas kinematics see Márquez et al. 2003). The slit positions (measured from North to East) are drawn on the figures, together with the bar sizes and ellipticities as determined in Márquez et al. (1999), for the primary bar in single-barred galaxies (NGC 6814 and NGC 6951) and for primary and secondary (inner) bars in double-barred ones (IC 184, NGC 2639 and UGC 3223). The results for the CaII triplet $E W$ measurements of each galaxy are presented in Table 3. Since we are interested in searching for the properties of stellar populations in the regions where the dips have been detected, the values given in Table 3 refer to the whole region occupied by the drops.

\subsection{IC 184}

The rotation curves along $\mathrm{PA}=7^{\circ}$ (major axis) (Fig. 1) and $97^{\circ}$ show that the rotation of the gas and stars are strongly decoupled along $\mathrm{PA}=7^{\circ}$ within \pm 4 arcsec of the center, with a central region of stellar counter-rotation within 1 arcsec, which is also visible along $\mathrm{PA}=97^{\circ}$ (not shown here). In order to know if such kinematics are due to the presence of a counter rotating core, as it is the case for NGC 4621 (Wernli et al. 2002) integral field spectroscopy is required. A small dip of about $20 \mathrm{~km} \mathrm{~s}^{-1}$ with respect to the maximum value is observed in the velocity dispersion in the innermost \pm 2 arcsec. The stellar velocity distribution along $\mathrm{PA}=97^{\circ}$ shows structure within the innermost \pm 9 arcsec, with a wavy central shape similar to that observed along $\mathrm{PA}=7^{\circ}$. This extension corresponds to that of the primary bar, which extends along $\mathrm{PA}=170^{\circ}$ (Márquez et al. 2000), i.e. only $17^{\circ}$ from the disk major axis. We note that the secondary bar extends \pm 4 arcsec along $\mathrm{PA}=30^{\circ}$ (Márquez et al. 2000) and therefore the velocity difference between the stellar and gas components in this region is most likely due to the non-circular motions caused by the secondary bar. $E W(\mathrm{CaT})$ shows a slight tendency for central values to be higher along both PAs, particularly along PA $=97^{\circ}$ (Fig. 6a). 

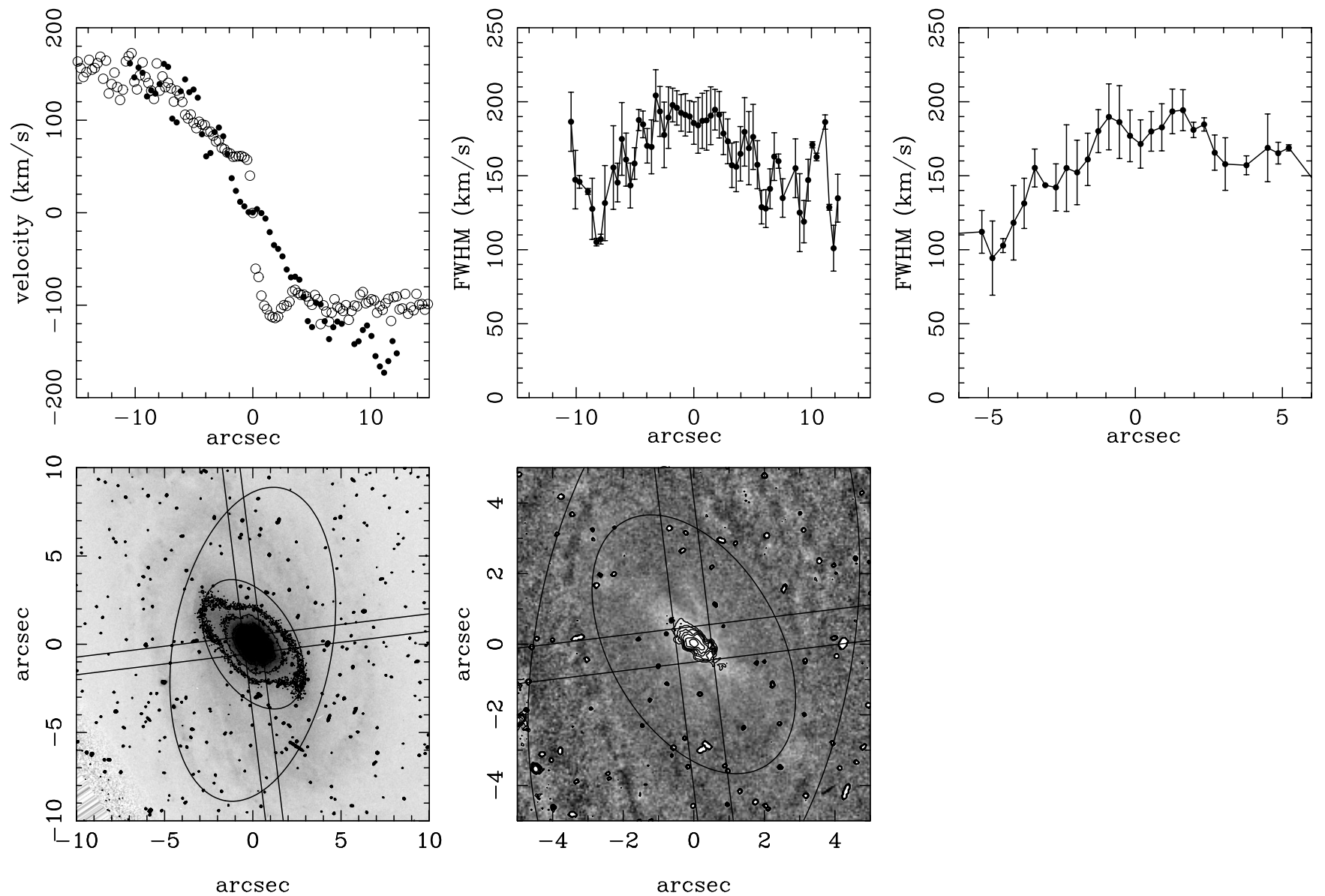

Fig. 1. IC 184. Top left: velocity curve of the gas (open circles, from Márquez et al. 2003) and stars (black circles) along PA $=7^{\circ}$. Top middle: $F W H M$ of the stellar component along $\mathrm{PA}=7^{\circ}$. Top right: $F W H M$ of the stellar component along PA $=97^{\circ}$. Bottom left: HST image of IC 184 in the F606W band with the two slits superimposed; the ellipses correspond (PA and ellipticities) to the two bars detected in the infrared (Márquez et al. 1999). Bottom middle: sharp divided image of the center.

The HST image of IC 184 (Fig. 1) shows a structure in the innermost 1 arcsec elongated along $\mathrm{PA} \approx 50^{\circ}$, very close to the position angle of the inner bar in the same image $\left(\mathrm{PA} \approx 40^{\circ}\right.$ ); although this feature is detected in a much smaller region, its PA is consistent with that of the small inner bar already detected in the infrared (Márquez et al. 1999). A structure resembling two straight dust lanes may be seen in the sharp-divided image running along about $\mathrm{PA} \approx 45^{\circ}$, below the center to the SW and above the center to the NE, respectively.

\subsection{UGC 3223}

The rotation curve of UGC 3223 along $\mathrm{PA}=80^{\circ}$ (very close to the bar major axis, $\mathrm{PA}=75^{\circ}$ ), although rather noisy, shows that the overall rotation of the gas follows, within the error bars, that of the stars (Fig. 2). Positive stellar velocities are systematically lower (down to $80 \mathrm{~km} \mathrm{~s}^{-1}$ ) than their negative counterparts for radii between 2 and 20 arcsec. A small dip $\left(\approx 10 \mathrm{~km} \mathrm{~s}^{-1}\right)$ may be observed in the velocity dispersion in the innermost \pm 1 arcsec, but needs to be confirmed with data of higher signal to noise. In spite of the low $\mathrm{S} / \mathrm{N}$ ratio of the spectra, an increase in
$E W(\mathrm{CaT})$ is detected in the innermost \pm 1 arcsec, corresponding to the extension of the detected dip (see Fig. 6b).

The HST image of UGC 3223 (Fig. 2) shows an asymmetric structure in the innermost 1 arcsec elongated mainly along $\mathrm{PA}=80^{\circ}$, and more extended to the West, consistent with that of the small inner bar already detected in the infrared (Márquez et al. 1999). Another extension can be seen to the NE along $\mathrm{PA} \sim 55^{\circ}$, resembling what could be an incomplete ring at $r=0.9$ arcsec.

\subsection{NGC 2639}

NGC 2639 is a galaxy optically classified as unbarred, but barred at NIR wavelengths (Márquez et al. 1999). The stellar and gas rotation curves of NGC 2639 along PA $=135^{\circ}$ (disk and main bar major axis, not shown here) show that both components have different velocities (up to $100 \mathrm{~km} \mathrm{~s}^{-1}$ ) in the region inside the bar (inner \pm 8 arcsec) (Márquez et al. 2003). The gaseous velocity distribution along $\mathrm{PA}=0^{\circ}$ shows departures of $50 \mathrm{~km} \mathrm{~s}^{-1}$ from pure rotation in the region within $r<4 \operatorname{arcsec}$ (Márquez et al. 2003). Along $\mathrm{PA}=45^{\circ}$ the velocity distribution is quite flat, as expected along a position 

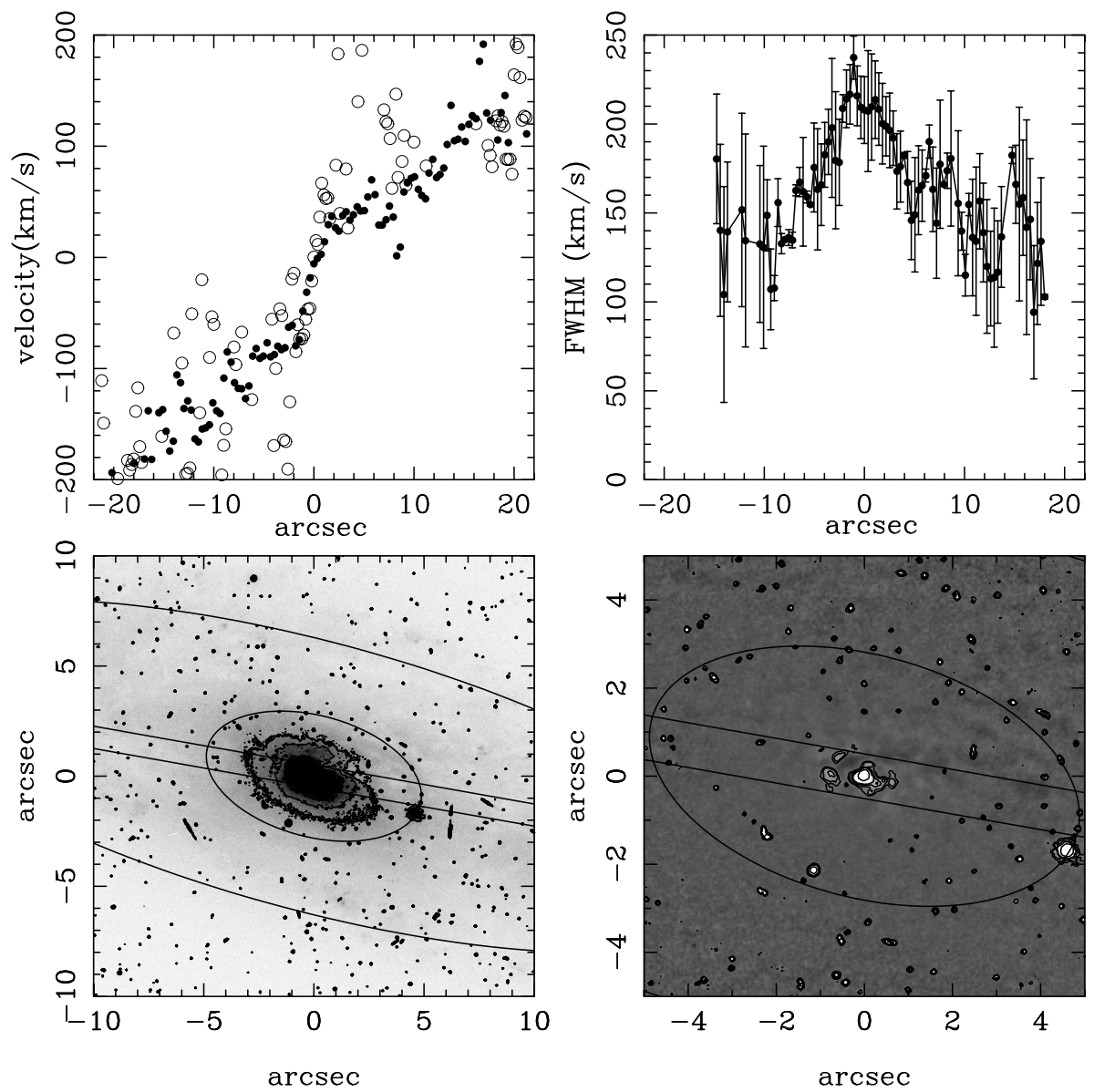

Fig. 2. UGC 3223. Top left: velocity curve of the gas (open circles, from Márquez et al. 2003) and stars (black circles) along PA $=80^{\circ}$. Top right: $F W H M$ of the stellar component along PA $=80^{\circ}$. Bottom left: HST image of UGC 3223 in the F606W band with the two slits superimposed; the ellipses correspond (PA and ellipticities) to the two bars detected in the infrared (Márquez et al. 1999). Bottom right: sharp divided image of the center.

angle close to the minor axis, whereas clear non-circular motions are traced by the gas kinematics in the central 2-3 arcsec radius (Fig. 3). A velocity dispersion drop of $\approx 20 \mathrm{~km} \mathrm{~s}^{-1}$ is detected along the two PAs in the innermost \pm 2 arcsec, being more clearly detected along PA $=45^{\circ}$ (Fig. 3). A hint of an increase in $E W(\mathrm{CaT})$ is detected along $\mathrm{PA}=45^{\circ}$ and $135^{\circ}$ (Fig. 6c).

The HST image of NGC 2639 (Fig. 3) shows a structure elongated along $\mathrm{PA}=135^{\circ}$ in the innermost \pm 1 arcsec (consistent with the thin inner bar detected in the infrared, Márquez et al. 1999), which is somewhat more extended and curved towards the NW.

\subsection{NGC 6814}

NGC 6814 has a small inclination and it is therefore difficult to derive its kinematical parameters accurately. It is a single barred galaxy, with a \pm 12 arcsec bar extending along PA $=25^{\circ}$ (Márquez et al. 1999). Note that the innermost \pm 3 arcsec show velocity dispersions about $30 \mathrm{~km} \mathrm{~s}^{-1}$ lower than the average values out to about 8 arcsec along $\mathrm{PA}=120^{\circ}$, an effect which also seems to be detected along $\mathrm{PA}=30^{\circ}$. Higher $\mathrm{S} / \mathrm{N}$ data are needed in order to confirm the presence of this drop. Although noiser, this profile resembles that of NGC 6951, both showing a broader central drop than the other three galaxies in our sample. As shown in Fig. 6d, a slight enhancement in $E W(\mathrm{CaT})$ has been detected along the extension of the dip along $\mathrm{PA}=30^{\circ}$.

The HST sharp-divided image of NGC 6814 shows a small $(r<2$ arcsec) several-armed spiral structure (in dark grey), with a clear elongation to the West along $\mathrm{PA}=120^{\circ}$ in the innermost 1.5 arcsec and a dust lane along $\mathrm{PA} \approx 60^{\circ}$ within $1 \operatorname{arcsec}$ (Fig. 4).

\subsection{NGC 6951}

The stellar velocity distributions of NGC 6951 are given in Pérez et al. (2000). We recall that two stellar components have been clearly detected, with amplitude differences up to $50 \mathrm{~km} \mathrm{~s}^{-1}$ along $\mathrm{PA}=84^{\circ}$ and $\mathrm{PA}=138^{\circ}$. A similar behavior is observed along PA $=48^{\circ}$ in the region between $r=5$ and 10 arcsec, which corresponds to stellar velocity dispersion peaks (Fig. 5). Higher S/N data would be required to obtain FWHM measurements for the two separate components, but their physical connection with the $F W H M$ peaks seems to be hinted by our data. The enhancement of $E W(\mathrm{CaT})$ in the 

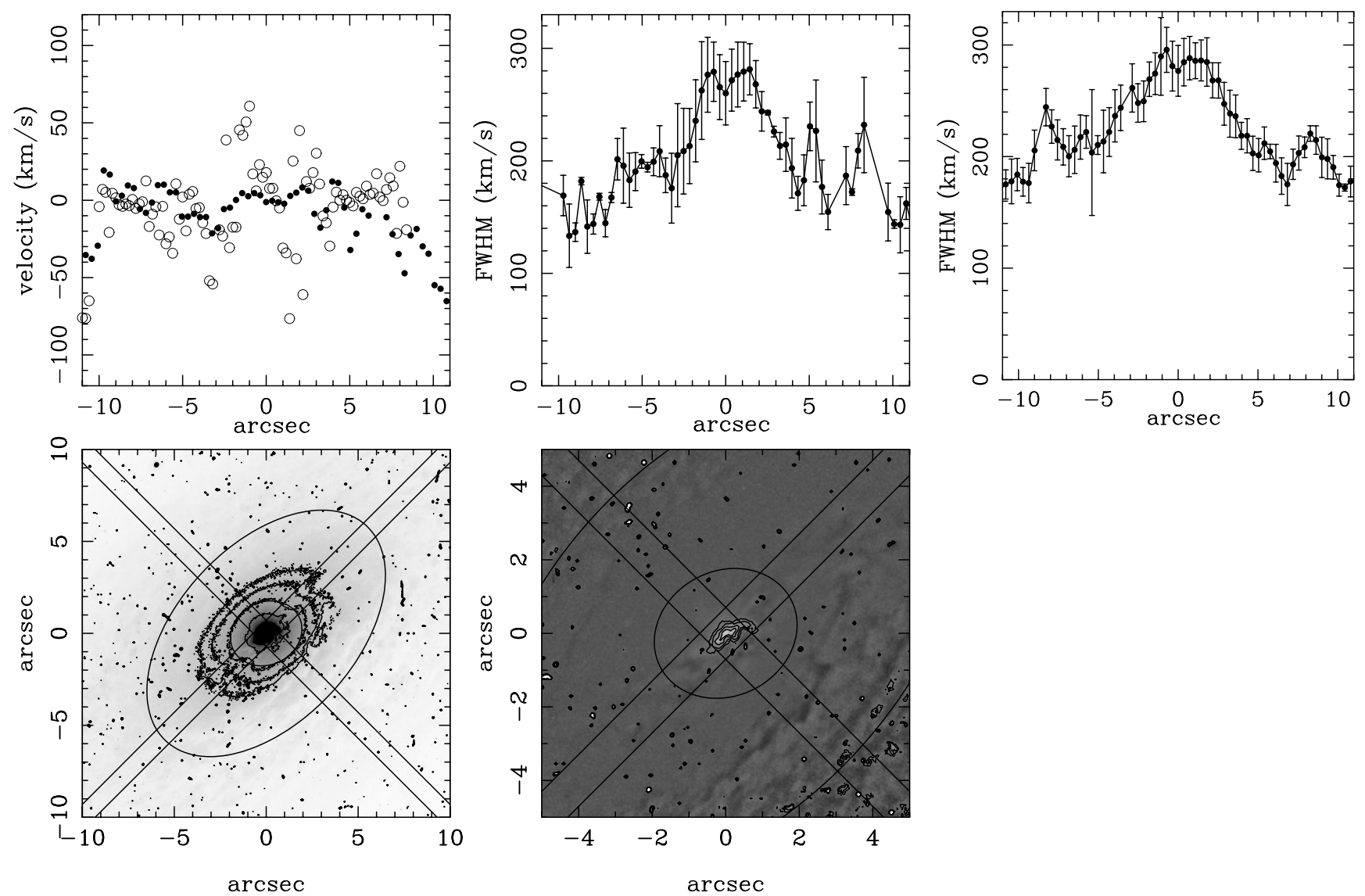

Fig. 3. NGC 2639. Top left: velocity curve of the gas (open circles, from Márquez et al. 2003) and stars (black circles) along PA $=45^{\circ}$. Top middle: $F W H M$ of the stellar component along PA $=45^{\circ}$. Top right: $F W H M$ of the stellar component along PA=135 . Bottom left: $\mathrm{HST}$ image of NGC 2639 in the F606W band with the two slits superimposed; the ellipses correspond (PA and ellipticities) to the two bars detected in the infrared (Márquez et al. 1999). Bottom middle: sharp divided image of the center.

central region was already reported and discussed in Pérez et al. (2000).

\section{Discussion and conclusions}

New stellar kinematics for a sample of four active galaxies using the Calcium triplet have been obtained and we have shown that reliable stellar velocity curves can be traced. Three of these galaxies show a double bar-like confirmed structure at optical and/or near IR frequencies. The previously reported data for NGC 6951 (a single-barred galaxy with a nuclear spiral, Pérez et al. 2000) have been added to the present study, in particular the velocity dispersion curve.

We have observed a dip in the stellar velocity dispersion in the innermost $\pm(1-3)$ arcsec of these five active galaxies based on observations made with 4 meter class telescopes. It is worth noticing that the velocity dispersion drops have been detected in three double-barred galaxies and in two single-barred ones, already indicating that the presence of nested bars is not a necessary condition; the drops in the later cases seem to be broader than in the former.

Note that such a dip is until now a rarely observed phenomenon, reported so far only for eleven nearby galaxies (most of them listed in Wozniak et al. 2003b; see references below), three of them from recent VLT observations using the ${ }^{12} \mathrm{CO}$ bands in the near IR (Emsellem et al. 2001). This is most probably due to the difficulty of estimating the $F W H M$ of the stellar component with a sufficient signal to noise ratio and spatial resolution.

Michel-Dansac \& Wozniak (2003) have recently modeled the evolution of isolated galaxies over several Gyrs using a selfconsistent $N$-body code including stars, gas and star formation. With such models, they succeeded in accounting for the drop in velocity dispersion observed by Emsellem et al. (2001) in three double-barred and one single-barred active galaxies, with a young stellar population born from dynamically cold gas which is accreted in a circumnuclear disk and creates the dip (Wozniak et al. 2003a; Wozniak \& Michel-Dansac 2003). We note that their simulations do not require the presence of embedded bar structures, since such processes can be induced by the primary bar itself (see also Wozniak et al. 2003b).

Since under these circumstances a luminosity dominating young stellar component is expected in the region where the drops are observed, it is worth trying to detect such a component in order to better validate the model. We have attempted to do so by analyzing the equivalent widths of the CaT lines along the dips and by inspecting the inner structure in high 

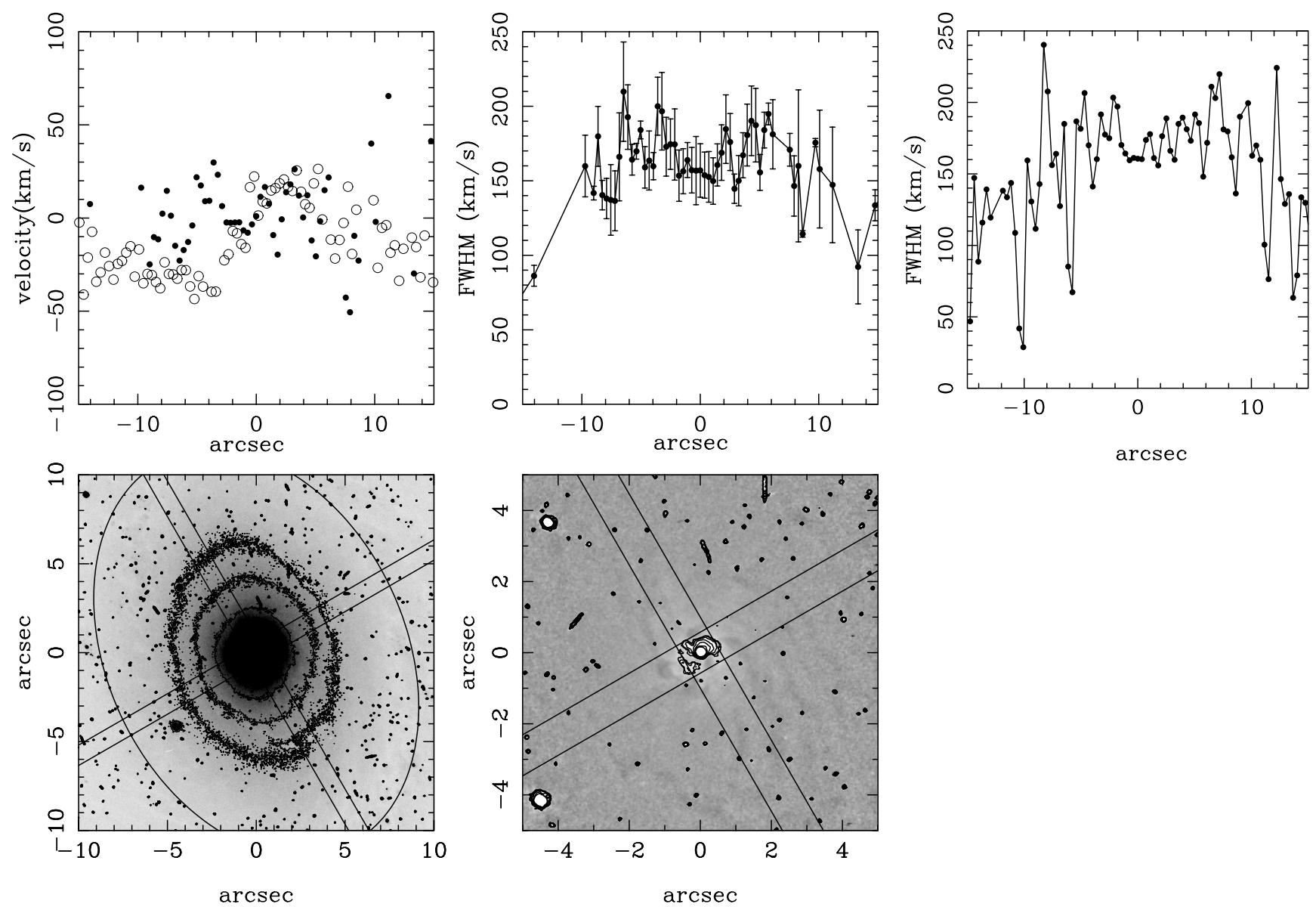

Fig. 4. NGC 6814. Top left: velocity curve of the gas (open circles, from Márquez et al. 2003) and stars (black circles) along PA =120\%. Top middle: $F W H M$ of the stellar component along PA $=120^{\circ}$. Top right: $F W H M$ of the stellar component along PA $=30^{\circ}$. Bottom left: HST image of NGC 6814 in the F606W band with the two slits superimposed; the ellipse corresponds (PA and ellipticity) to the bar detected in the infrared (Márquez et al. 1999). Bottom middle: sharp divided image of the center.

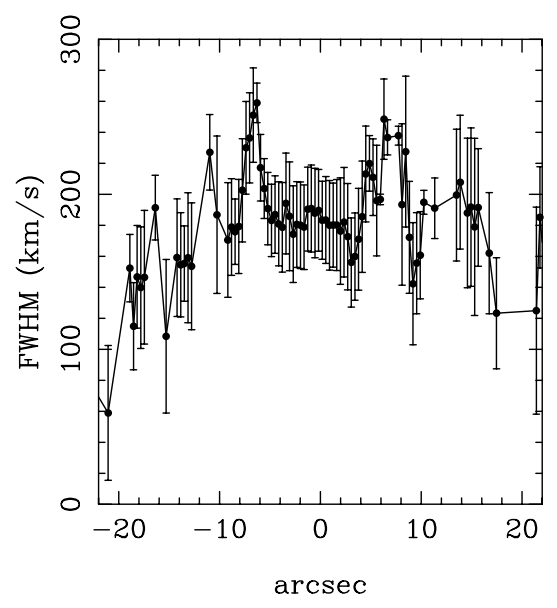

Fig. 5. NGC 6951. FWHM of the stellar component along $\mathrm{PA}=48^{\circ}$.

resolution HST images. The analysis of HST images for the target galaxies shows that structure is indeed present in the regions where the dips occur in IC 184, UGC 3223 and NGC $2639^{4}$.

\footnotetext{
${ }^{4}$ With our small sample (5 galaxies) we find no dependence of the drop sizes with respect to those of the outer/inner bars; a larger sample should be needed in order to analyze this point.
}

The same conclusion is valid when inspecting the sharpdivided frames of those galaxies with available HST archive images and with velocity dispersion drops reported in the literature, namely NGC 1365 and NGC 1808 (Emsellem et al. 2001; for the Sy1 NGC 1097 there are only F218W images, that do not allow to detect such structures), NGC 6503 (Bottema 1993), NGC 3627, NGC 4303, NGC 4579 (Héraudeau \& Simien 1998), NGC 4725 (Héraudeau et al. 1999; diffraction spikes impede a reliable determination of the hinted central elongated structure), NGC 4477 (Jarvis et al. 1988), NGC 3623 (de Zeeuw et al. 2002) and NGC 3412 (Aguerri et al. 2003). Note that the images are at a wavelength range that does not include the CaT, so we do not expect to find exactly the same structure. Also the spectroscopic structure is diluted because of the poorer ground-based spatial resolution. Therefore, the presence of structure in HST images at the scales of the velocity dispersion drops is only a consistency check. The existence of a direct correlation between the two observables needs further investigation with similar spatial resolution data.

The velocity dispersion drop in NGC 6814 seems to be as broad as that in NGC 6951, and it is only for NGC 6814 and NGC 6951 (LINER/Sy2) that the drops seem to be present with no associated elongation in the images. This may indicate that 

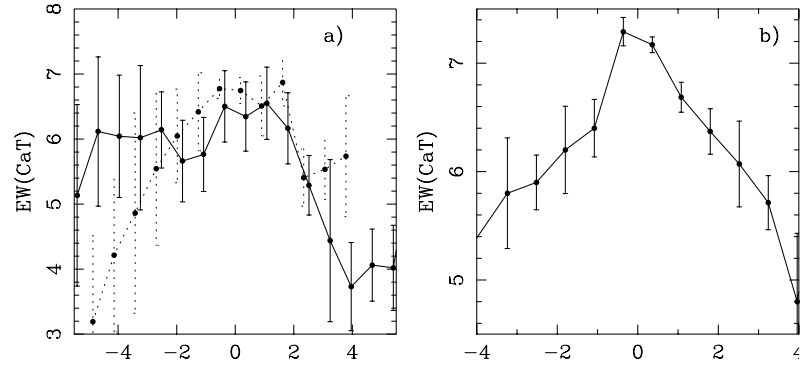

$\operatorname{arcsec}$
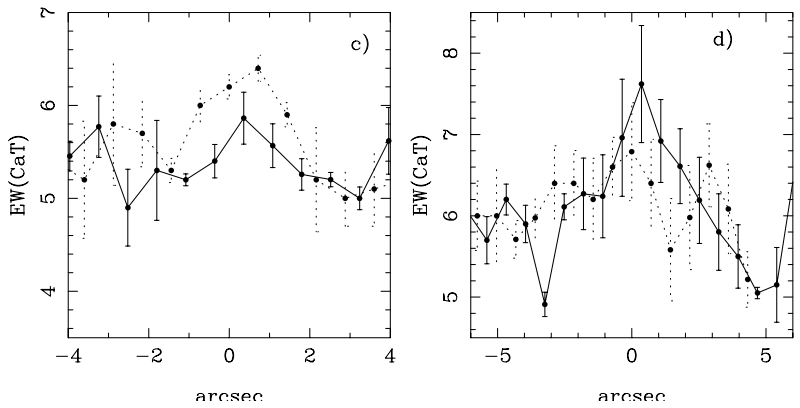

Fig. 6. Spatial distribution of the CaT equivalent width $(E W)$ in the central regions of: a) IC $184\left(\mathrm{PA}=7^{\circ}\right.$ as full line, $\mathrm{PA}=97^{\circ}$ as dotted line), b) UGC $3223, \mathrm{PA}=80^{\circ}$, c) NGC $2639\left(\mathrm{PA}=45^{\circ}\right.$ as full line, $\mathrm{PA}=135^{\circ}$ as dotted line $)$ and d) NGC $6814\left(\mathrm{PA}=30^{\circ}\right.$ as full line, $\mathrm{PA}=120^{\circ}$ as dotted line). The corresponding plot for NGC 6951 is given in Pérez et al. (2000).

such structures either are seen face-on in these cases or are absent. For the other galaxies, if the elongations are interpreted as intrinsically circular disks, these disks would be placed almost perpendicular to the main disk in NGC $2639\left(i_{\text {inner disk }} \approx 74^{\circ}\right)$, at intermediate angle for IC $184\left(i_{\text {inner disk }} \approx 40^{\circ}\right)$ and coplanar with the main disk in UGC 3223.

The calcium triplet equivalent width, $E W(\mathrm{CaT})$, is expected to increase for younger populations. Unfortunately, the comparison with stellar population models to test the age of such a population is rather risky, due to the already mentioned problem with the determination of the continuum bands which impedes a quantitative comparison with other authors (for instance, the models developed by García-Vargas et al. 1998 require $E W \mathrm{~s}$ higher than those we have measured to invoke an important contribution of super-giant stars). The variations on the values of the $E W$ s can still be compared but, due to the accuracy achievable by using $E W(\mathrm{CaT})$ measurements on our data, the analysis is not straightforward since we expect the relative differences in the $E W(\mathrm{CaT})$ to be comparable to our accuracy (from 1 to $1.5 \AA$ ), and consequently such variations can, strictly speaking, correspond to a constant value. Nevertheless, a hint of a local enhancement in the drop region is suggested by the data, a behavior that could be due to the presence in these regions of a population different from that of the bulge.

To confirm or discard Wozniak et al.'s models the analysis of higher $\mathrm{S} / \mathrm{N}$ spectra in a much broader wavelength range is needed to constrain the age of the observed stellar populations detected in the central dips. On this respect, we notice that some of the galaxies show evidence for the presence of an intermediate (NGC 3627 and NGC 6503, González Delgado et al., in preparation) or young (NGC 4303, Colina et al. 2002) population in the regions where the dispersion drops take place and where elongations are seen in the HST sharp-divided images.

The case of NGC 6951 is especially interesting since an alternative explanation for the presence of the apparent drop within $r=5$ arcsec in this galaxy would be the existence of two stellar kinematic components. This appears as a possibility to be checked in the other four galaxies with higher spectral resolution and $\mathrm{S} / \mathrm{N}$ data. It would therefore be of particular interest to re-observe these five galaxies with an 8 meter class telescope, to define with better precision the characteristics of the dip, in particular its disk nature (for which several position angles are required), and also to observe a larger sample of galaxies with and without an AGN. Note that among the 16 galaxies with reported drops -5 of them presented for the first time in this work -15 host $\mathrm{AGNs}^{5}$, either Seyfert 1 (3), Seyfert 2 (7), LINERs (2), or intermediate type LINER/Sy2 (3); the only galaxy with no evident signs of nuclear activity is NGC 3412 (Ho et al. 1995). The DEGAS sample is particularly well adapted for this purpose, since it is well defined and comprises only isolated galaxies, where the complicating effects of galaxy interactions are not present.

Acknowledgements. We thank the referee, Eric Emsellem, for his excellent report, which has largely helped us improve the clarity of the paper. I.M. acknowledges financial support from the IAA and the Spanish Ministerio de Ciencia y Tecnología through a Ramón y Cajal fellowship. This work is financed by DGICyT grants PB93-0139, PB96-0921, PB98-0521, PB98-0684, ESP981351, AYA2001-2089, AYA2001-3939-C03-01 and the Junta de Andalucía grant TIC-144. We acknowledge financial support from the Picasso Programme d'Actions Integrées of the French and Spanish Ministries of Foreign Affairs. F.D. acknowledges financial support from CNRS-INSU for several observing trips. E.P. acknowledges financial support from the CONACYT (México) grant 36132-E. J. Maza gratefully acknowledges support from the Chilean Centro de Astrofísica FONDAP 15010003. We acknowledge Ignacio Marrero for his collaboration in some data acquisition and calibration. This research has made use of the NASA/IPAC extragalactic database (NED), which is operated by the Jet Propulsion Laboratory under contract with the National Aeronautics and Space Administration.

\section{References}

Aguerri, J. A. L., Debattista, V. P., \& Corsini, E. M. 2003, MNRAS, 338,465

Barnes, J. E., \& Hernquist L. E. 1991, ApJ, 370, 65

Bottema, R. 1993, A\&A, 275, 16

Cenarro, A. J., Cardiel, N., Gorgas, J., et al. 2001, MNRAS, 326, 959

Colina, L., González Delgado, R. M., Mas-Hesse, J. M., Leitherer, C., \& Jiménez-Bailón, E. 2002, ApJ, 579, 545

Combes, F. 1994, in Mass Transfer Induced Activity in galaxies (Cambridge University Press), 170

Emsellem, E., Greusard, D., Combes, F., et al. 2001, A\&A, 368, 52

Erwin, P., \& Sparke, L. 1999, ApJ, 521, 37

Erwin, P., \& Sparke, L. S. 2002, AJ, 124, 65

Friedli, D., \& Martinet, L. 1993, A\&A, 277, 27

5 Their nuclear classification has been taken from NED and is given in Table 2. 
García-Vargas, M. L., Mollá, M., \& Bressan, A. 1998, A\&AS, 130, 513

Heckman, T. M. 1980, A\&A, 87, 142

Heller, C. H., \& Shlosman, I. 1994, ApJ, 424, 84

Héraudeau, Ph., \& Simien, F. 1998, A\&AS, 133, 317

Héraudeau, Ph., Simien, F., Maubon, G., \& Prugniel, Ph. 1999, A\&AS, 136, 509

Ho, L. C., Filippenko, A. V., \& Sargent, W. L. 1995, ApJS, 98, 477

Jarvis, B. J., Dubath, P., Martinet, L., \& Bacon, R. 1988, A\&AS, 74, 513

Knapen, J. H., Shlosman, I., \& Peletier, R. F. 2000, ApJ, 529, 93

Jogee, S., Kenney, J. D. P., \& Smith, B. J. 1999, ApJ, 526, 665

Laine, S., Knapen, J. H., Pérez-Ramírez, D., Doyon, R., \& Nadeau, D. 1999, MNRAS, 302, L33

Laine, S., Shlosman, I., Knapen, J. H., \& Peletier, R. 2002, ApJ, 567, 97

Maiolino, R., Alonso-Herrero, A., Anders, S., et al. 2000, ApJ, 531, 219

Márquez, I., \& Moles, M. 1996, A\&AS, 120, 1

Márquez, I., Durret, F., González Delgado, R. M., et al. 1999, A\&AS, 140,1

Márquez, I., Durret, F., Masegosa, J., et al. 2000, A\&A, 360, 431

Márquez, I., Masegosa, J., Moles, M., et al. 2002, A\&A, 393, 389

Márquez, I., Durret, F., Masegosa, J., et al. 2003, in preparation

Martini, P., \& Pogge, R. W. 1999, AJ, 118, 2646

Martini, P., Regan, M. W., Mulchaey, J. S., \& Pogge, R. W. 2003a, ApJS, 146, 353
Martini, P., Regan, M. W., Mulchaey, J. S., \& Pogge, R. W. 2003b, ApJ, 589, 774

McLeod, K. K., \& Rieke, G. H. 1995, ApJ, 441, 96

Michel-Dansac, L., \& Wozniak, H. 2003, Proc. Symp., The evolution of galaxies. III. From simple approaches to self-consistent models, Kiel, Germany, July 16-20, 2002

Moles, M., Márquez, I., \& Pérez, E. 1995, ApJ, 438, 604

Mulchaey, J. S., \& Regan, M. W. 1997, ApJ, 482, L135

Pérez, E., Márquez, I., Marrero, I., et al. 2000, A\&A, 353, 893

Regan, M. W., \& Mulchaey, J. S. 1999, AJ, 117, 2676

Shlosman, I., Frank, J., \& Begelman, M. 1989, Nature, 338, 45

Shlosman, I., Peletier, R. F., \& Knapen, J. H. 2000, ApJ, 565, 921

Simkim, S. M., Su, H. J., \& Schwarz, M. P. 1980, ApJ, 237, 404

Sofue, Y., Yoshida, S., Aoki, T., et al. 1994, PASJ, 46, 1

Terlevich, E., Díaz, A. I., \& Terlevich, R. 1990, MNRAS, 242, 271

Wernli, F., Emsellem, E., \& Copin, Y. 2002, A\&A, 396, 73

Wozniak, H., \& Michel-Dansac, L. 2003, Proc. Symp., The evolution of galaxies. III. From simple approaches to self-consistent models, Kiel, Germany, July 16-20, 2002

Wozniak, H., Combes, F., Emsellem, E., et al. 2003a, Proc. Symp., Active galactic nuclei, from central engine to host galaxy, Observatoire de Paris-Meudon July 23-27, 2002

Wozniak, H., Combes, F., Emsellem, E., \& Friedli, D. 2003b, A\&A, in press

de Zeeuw, T., Bureau, M., Emsellem, E., et al. 2002, MNRAS, 329,513 